ISSN 2075-1680

www.mdpi.com/journal/axioms

\title{
Communication
}

\section{On Transcendental Numbers}

\section{Florin F. Nichita}

Simion Stoilow Institute of Mathematics of the Romanian Academy, 21 Calea Grivitei Street, Bucharest 010702, Romania; E-Mail: Florin.Nichita@ imar.ro; Tel: +40-21-319-65-06; Fax: +40-21-319-65-05

Received: 13 December 2013; in revised form: 7 February 2014 / Accepted: 19 February 2014 / Published: 21 February 2014

\begin{abstract}
Transcendental numbers play an important role in many areas of science. This paper contains a short survey on transcendental numbers and some relations among them. New inequalities for transcendental numbers are stated in Section 2 and proved in Section 4. Also, in relationship with these topics, we study the exponential function axioms related to the Yang-Baxter equation.
\end{abstract}

Keywords: transcendental numbers; Yang-Baxter equation; inequalities; exponential function; computational methods; standard distribution

Classification: MSC 16T25; 11D09; 11D25; 11N45; 33B10; 60A05; 65L70

\section{Introduction}

In [1], the author considers two types of scientists, and mathematics needs both of them. Birds fly high, and, therefore, they can see the whole landscape; they resemble scientists who try to unify theories, who obtain important results, and who have a broad understanding. As an example, among others, the author considers Chen Ning (Frank) Yang. On the other hand, the scientists who resembles frogs work on problems which are less influential. Mathematics is rich and beautiful because birds give it broad visions and frogs give it intricate details.

In a similar manner, Solomon Marcus ([2]) used the terms "bees" versus "ants" in his talks, describing mathematicians who are involved in many different areas of research versus the mathematicians who work on a restricted domain.

A transdisciplinary approach (see [3,4]) attempts to discover what is between disciplines, across different disciplines, and beyond all disciplines. The algebraic model for transdisciplinarity from [5] explains how disciplinarity, interdisciplinarity, pluridisciplinarity and transdisciplinarity are related. 
Because there is a huge number of new disciplines, it is important to have a transdisciplinary understanding of the world.

Our paper is written in transdisciplinary fashion, because important transcendental numbers play a role in many areas of science. We use results and concepts from algebra, mathematical analysis, probability and statistics, computer science, numerical analysis, etc. The next section is a short survey on transcendental numbers and some relations among them; new inequalities are stated. In Section 3, we study the exponential function axioms related to the Yang-Baxter equation. Section 4 deals with proofs and approximations of the number $\pi$ (see also [6,7]). In our last section we state a new inequality, and we argue that computer science plays an important role in the development of the modern mathematics.

\section{Transcendental Numbers, Famous Relations and New Inequalities}

It is well-known the relation which contains the numbers $e, \pi$ and $i$ :

$$
e^{\pi i}=-1
$$

It follows that: $\quad e^{\pi}=\left(e^{i \pi}\right)^{-i}=(-1)^{-i} ; \quad i^{i}=\left(e^{\frac{i \pi}{2}}\right)^{i}=e^{-\frac{\pi}{2}}$.

Another famous relation between $e$ and $\pi$ is the following:

$$
\int_{-\infty}^{\infty} e^{-x^{2}} d x=\sqrt{\pi}
$$

The above formula plays an important role in probability and statistics. It can be read as the area of the subgraph for the standard distribution. The proof of the above formula follows from using some double integrals and a change of variables to polar coordinates (see, for example, [8]).

The inequality for real numbers

$$
x^{2}+e>\pi x \quad \forall x
$$

provides a new relation between the transcendental numbers $e$ and $\pi$. It can be restated as an approximation for $\pi$. Also, it is related to a more complicated inequality for real numbers:

$$
x^{2}>\frac{\sqrt{2} x-\sqrt{3}}{x^{2}-\pi x+e} \quad \forall x
$$

Of course, one has to prove our first inequality in order to make sure that the denominator of the fraction appearing in our second inequality is different from zero.

Several inequalities emerge:

$$
\begin{gathered}
\cos (e)<\sin \left(\frac{6 \pi}{5}\right) \\
4 \log _{\pi} e+e \ln \pi \quad>2 \pi \\
\left|e^{1-z}+e^{\bar{z}}\right|>\pi \quad \forall z \in \mathbb{C}
\end{gathered}
$$

in the last inequality if $z=-i$, we have: $\left|e^{i}+e^{1+i}\right|>\pi$.

These inequalities will be (proved and) studied in our last section. 


\section{The Yang-Baxter Equation}

The Equation (1) can be generalized for matrices. This will be explained at the end of this section.

In our special issues on Hopf algebras, quantum groups and Yang-Baxter equations, several papers [9-16], as well the feature paper [17], covered many topics related to the Yang-Baxter equation. The Yang-Baxter equation was solved only in dimension 2, using computational methods.

The terminology of this section is compatible with the above cited papers, and the constructions which follow are related to the paper [18], and to the formula (1). To our knowledge this point of view (and construction) is new.

Let $V$ be a complex vector space, and $I_{j}: V^{\otimes j} \rightarrow V^{\otimes j} \quad \forall j \in\{1,2\}$ identity maps.

We consider $J: V^{\otimes 2} \rightarrow V^{\otimes 2}$ a linear map which satisfies

$J \circ J=-I_{2} ; \quad J^{12} \circ J^{23}=J^{23} \circ J^{12}$, where $J^{12}=J \otimes I_{1}, \quad J^{23}=I_{1} \otimes J$.

Then, $R(x)=\cos x I_{2}+\sin x J$ satisfies the colored Yang-Baxter equation:

$$
R^{12}(x) \circ R^{23}(x+y) \circ R^{12}(y)=R^{23}(y) \circ R^{12}(x+y) \circ R^{23}(x)
$$

The proof of Equation (5) could be done directly. Another way to prove it is to write $R(x)=e^{x J}$ (it makes sense!), and to check that Equation (5) reduces to

$$
x J^{12}+(x+y) J^{23}+y J^{12}=y J^{23}+(x+y) J^{12}+x J^{23}
$$

For example, in dimension two, the matrix of $J$ could be:

$$
\left(\begin{array}{cccc}
0 & 0 & 0 & \frac{1}{\alpha} i \\
0 & 0 & i & 0 \\
0 & i & 0 & 0 \\
\alpha i & 0 & 0 & 0
\end{array}\right)
$$

In this case, the matrix form of $R(x)$ is the following:

$$
\left(\begin{array}{cccc}
\cos x & 0 & 0 & \frac{i}{\alpha} \sin x \\
0 & \cos x & i \sin x & 0 \\
0 & i \sin x & \cos x & 0 \\
\alpha i \sin x & 0 & 0 & \cos x
\end{array}\right)
$$

Remark 1. The Equation (1) can be rephrased for the above matrices as follows:

$$
e^{\pi J}=-I_{2}
$$

This matrix is a trivial solution for the constant Yang-Baxter equation. It would be interesting to relate it to the colored Yang-Baxter equation by using some Baxterization procedure. 


\section{Proofs for Our Inequalities}

Our approach to prove the inequalities from Section 2 is to consider the associated equations, and to prove that they have no real solutions. (My students at AUK University used the graphing calculators to solve some of them.)

Remark 2. The following equation has no real solutions: $x^{2}-\pi x+e=0$.

Indeed, one could use the quadratic formula and two digit approximations for $e$ and $\pi$ to prove that $\Delta=\pi^{2}-4 e<0$. The inequality

$$
\pi<2 \sqrt{e}
$$

is an approximation of $\pi$ (see, also, [6]).

Some applications of this result could be in probability and statistics. (See formula (2)) Thus, one can find an upper bound for the area of the subgraph of the standard distribution over a certain interval $[a, b]$. If $\frac{\pi}{2}$ is an interior point (this idea could be developed further) of [a, b], then our approximation is efficient: $\quad \int_{a}^{b} e^{-x^{2}} d x<\left.\frac{1}{\pi} e^{u}\right|_{e-\pi b} ^{e-\pi a}$.

Remark 3. Solving an equation by using the quadratic formula and the graphing calculator is not always possible. For example, the following equation needs to be solved by another method:

$$
12345678 x^{2}+99999999 x+87654321=0
$$

One can use the formula $A x^{2}+(A+C) x+C=(A x+C)(x+1)$, for $A=12345678$ and $C=87654321$. The solution $x=-1$ could be observed directly. The other solution has to be expressed as a fraction in the simplest form (which is a tricky problem again).

Remark 4. The following equations have no real solutions:

(i) $\quad x^{4}-\pi x^{3}+e x^{2}-\sqrt{2} x+\sqrt{3}=0$;

(ii) $\quad x^{6}-\pi x^{5}+e x^{4}-\sqrt{2} x^{3}+\sqrt{3} x^{2}-\sqrt{5} x+\sqrt{13}=0$.

This can be checked on a graphing calculator. (What kind of computational methods could be considered for solving these equations?) The first of these equations can be stated as (4).

Is it possible to solve these equations algebraically? Recall that for equations of degree 6 there are no formulas for their solutions. We leave this questions as open problems.

The Equation (ii) can be restated as an inequality for real numbers, which could lead to approximations for $\pi$ :

$$
x+\frac{e}{x}-\frac{\sqrt{2}}{x^{2}}+\frac{\sqrt{3}}{x^{3}}-\frac{\sqrt{5}}{x^{4}}+\frac{\sqrt{13}}{x^{5}}>\pi \quad \forall x>0
$$

\section{Comments}

Remark 5. The following inequality (and approximation) is left without proof:

$$
\left|e^{i}-\pi\right|>e
$$

Remark 6. We think that the new problems presented in this paper could lead to other challenging ideas and questions. Are they pointing out to the fact that modern mathematics and computer science are 
dependent on each other? Are they leading to some kind of transdisciplinary approach? For what kind of problems from pure mathematics the computational methods are essential? Why pure mathematics cannot give solutions for those problems?

\section{Conflicts of Interest}

The author declares no conflict of interest.

\section{References}

1. Dysson, F. Birds and Frogs. Not. AMS 2009, 56, 212-223.

2. Meetings with Solomon Marcus; Spandonide, L., Paun, G., Eds.; Spandugino Publishing House: Bucharest, Romania, 2010.

3. Nicolescu, B. Manifesto of Transdisciplinarity; State University of New York (SUNY) Press: New York, NY, USA, 2002 ; translated in English by Karen-Claire Voss.

4. Nicolescu, B. Transdisciplinarity-past, present and future. In Moving Worldviews-Reshaping Sciences, Policies and Practices for Endogenous Sustainable Development; Haverkort, B., Reijntjes, C., Eds.; COMPAS: Leusden, The Netherlands, 2006; pp. 142-166.

5. Nichita, F.F. On Models for Transdisciplinarity. Transdiscipl. J. Eng. Sci. 2011, 2, 42-46.

6. Pi Approximations. Available online: http://mathworld.wolfram.com/PiApproximations.html (accessed on 20-02-2014).

7. Srivastava, H.M., Ed. q-Series and Related Topics in Special Functions and Analytic Number Theory. Axioms 2013, 2, 435-436.

8. Polar coordinate system. Wikipedia, the free encyclopedia. Available online: http://en.wikipedia.org/wiki/Polar_coordinate_system (accessed on 20-02-2014).

9. Kanakoglou, K. Gradings, Braidings, Representations, Paraparticles: Some Open Problems. Axioms 2012, 1, 74-98.

10. Underwood, R.G. Quasi-triangular Structure of Myhill-Nerode Bialgebras. Axioms 2012, 1, $155-172$.

11. Schmidt, J.R. From Coalgebra to Bialgebra for the Six-Vertex Model: The Star-Triangle Relation as a Necessary Condition for Commuting Transfer Matrices. Axioms 2012, 1, 186-200.

12. Nichita, F.F.; Zielinski, B. The Duality between Corings and Ring Extensions. Axioms 2012, 1, 173-185.

13. Links, J. Hopf Algebra Symmetries of an Integrable Hamiltonian for Anionic Pairing. Axioms 2012, 1, 226-237.

14. Hoffnung, A.E. The Hecke Bicategory. Axioms 2012, 1, 291-323.

15. Nichita, F.F. Yang-Baxter systems, algebra factorizations and braided categories. Axioms 2013, 2, 437-442.

16. Lebed, V. R-Matrices, Yetter-Drinfel'd Modules and Yang-Baxter Equation. Axioms 2013, 2, 443-476.

17. Nichita, F.F. Introduction to the Yang-Baxter Equation with Open Problems. Axioms 2012, 1, 33-37. 
18. Wang, G.; Xue, K.; Sun, C.; Du, G. Yang-Baxter R matrix, Entanglement and Yangian. Quantum Inf. Process. 2012, 11, 1775-1784.

(c) 2014 by the author; licensee MDPI, Basel, Switzerland. This article is an open access article distributed under the terms and conditions of the Creative Commons Attribution license (http://creativecommons.org/licenses/by/3.0/). 